\title{
Investigation of the UV Radiation Process in NGC 2023
}

\author{
Lee Dae-Hee \\ Korea Astronomy Observatory, 61-1 Hwaam-dong, Yusong-gu, Daejon \\ 305-348, Korea \\ Seon Kwang-Il \\ Korea Astronomy Observatory, 61-1 Hwaam-dong, Yusong-gu, Daejon \\ 305-348, Korea \\ Min Kyoung-Wook \\ Department of Physics, Korea Advanced Institute of Science and \\ Technology, 373-1 Gusong-dong, Yusong-gu, Daejon 305-701, Korea
}

\begin{abstract}
We observed the $\mathrm{H}_{2}$ absorption lines in the far- $\mathrm{UV}$ band toward HD 37903, which is the central star of the reflection nebula NGC 2023, and ran the radiative-collisional equilibrium program CLOUDY to simulate the UV radiation process of NGC 2023. The input parameters are chosen to generate the same ionization and excitation distribution of atoms and molecules as observed, so that we can create a physical model for NGC 2023.
\end{abstract}

\section{Introduction}

NGC 2023, a conspicuous reflection nebula in the southern part of the Orion B molecular cloud (L1630), is one of the most famous targets for studying PDRs. The central star, HD 37903, provides the principal illumination for NGC 2023, which forms an $\mathrm{H}$ II region and creates a PDR between the $\mathrm{H}$ II region and the rest of the neutral cloud. Many observations have been conducted in the various wavelength bands. $\mathrm{H}_{2}$ absorption lines are observed in the far-UV band toward HD 37903 (Lee et al. 2002; Meyer et al. 2001), while the $\mathrm{H}_{2}$ emission of NGC 2023 was studied by near-IR observation (Burton et al. 1998). Other species are also observed in the far-IR (Harvey, Thronson, \& Gatley 1980), radio (Gatley et al. 1987; Jaffe et al. 1990), and far-UV bands (Knauth et al. 2001). These observational results provide the clues how the UV radiation emitted from HD 37903 interacts with the surrounding gas and changes the physical conditions of NGC 2023. In this study, we run the radiative-collisional program CLOUDY (Ferland 1996) to simulate the UV radiation process of NGC 2023. By reproducing the same results as observed, we create a model for NGC 2023, which makes it possible to derive all the physical conditions of NGC 2023. 


\section{Analysis}

We obtain the $\mathrm{H}_{2}$ and $\mathrm{CO}$ column densities by analyzing the absorption profiles toward HD 37903 which was observed by the Berkeley Extreme and FarUltraviolet Spectrometer (BEFS) on the ORFEUS telescope in 1996. Table 1 shows the resultant column densities of the foreground cloud of HD 37903.

Table 1. $\mathrm{H}_{2}$ and $\mathrm{CO}$ column densities toward HD 37903 observed by BEFS

$\begin{array}{lccccccc}\text { Species } & N(0) & N(1) & N(2) & N(3) & N(4) & N\left(\mathrm{H}_{2}\right) & N(\mathrm{CO}) \\ \log \left(\mathrm{cm}^{-2}\right) & 20.6 \pm .0 & 20.4 \pm .0 & 19.7 \pm .1 & 18.6 \pm .1 & 17.2 \pm .6 & 20.9 \pm .0 & 14.4 \pm .5\end{array}$

For the CLOUDY model, we assume a plane parallel cloud. The ionization photons from the central hot star are absorbed to make an $\mathrm{H}$ II region between the star and the cloud. We set the hydrogen density of the surface of the cloud as $n=10^{5} \mathrm{~cm}^{-3}$, the UV intensity as 5000 times as higher than the ambient interstellar value, and the total $\mathrm{H}_{2}$ column density as $\log N\left(\mathrm{H}_{2}\right)=20.9$, so that we can reproduce each column density as observed. Table 2 shows the results of the model calculations by CLOUDY.

Table 2. $\mathrm{H}_{2}$ and $\mathrm{CO}$ column densities from the CLOUDY model

$\begin{array}{lccccccc}\text { Species } & N(0) & N(1) & N(2) & N(3) & N(4) & N\left(\mathrm{H}_{2}\right) & N(\mathrm{CO}) \\ \log \left(\mathrm{cm}^{-2}\right) & 20.5 & 20.3 & 19.5 & 18.5 & 17.3 & 20.8 & 13.9\end{array}$

\section{Concluding Remarks}

In this study, we obtain the column densities of each rotational state of $\mathrm{H}_{2}$ and $\mathrm{CO}$ by both in-orbit observations and CLOUDY model calculations. The computed physical parameters by model calculations are well consistent with the observed results. $\mathrm{H}_{2}$ fluorescent emission lines shall be plotted and compared with the observations.

\section{References}

Burton, M. G. et al. 1998, Publ. Astron. Soc. Austrailia, 15, 194

Ferland, G. J. et al. 1996, ApJ, 461, 683

Gatley, I. et al. 1987, ApJ, 318, L13

Harvey, P. M., Thronson, H. A. Jr., \& Gatley, I. 1980, ApJ, 235, 894

Jaffe, D. T. et al. 1990, ApJ, 353, 193

Knauth, D. C. et al. 2001, ApJS, 135, 201

Lee, D. H. et al. 2002, ApJ, 575, 234

Meyer, D. et al. 2001, ApJ, 535, L59 